\title{
Classification of Nasal Deviation
}

\author{
Abdul RS Juratli DLO-RCS Eng, FRCS (ORL-HNS) England 1,2* \\ ${ }^{1}$ Consultant ENT Surgeon University of Leicester, LRI Hospital, UK. 2004-2016 \\ ${ }^{2}$ Al Zahra Private Hospital, Sharjah, UAE. 2016 to date
}

*Corresponding author: Abdul R.S. Juratli, Consultant ENT Surgeon, Al Zahra private hospital, Sharjah, UAE.

Received Date: January 03, 2019

Published Date: January 25, 2019

\section{Abstract}

Due to its central location, the nose plays a prominent role in addressing the central facial axis which helps at camouflaging a large proportion of facial asymmetry. It gives the standard norm to the facial expressions, any little nasal deviation result in conspicuous facial disharmony and are often attended by nasal airway disfunction. The nasal deviation causes a cosmetic deformity that is often disconcerting for patients. Nasal deviation carries considerable risk at the nasal airflow by increasing the nasal valve resistance at the level of mid vault and/or lower alar structures.

The lack of objective measurement of the external nasal deviation puts the professions at risk of standardizing the severity of the deviation. It deprives the nasal plastic surgeons of having a common thought about the most likely convenient and available techniques for correcting the deviation in upper, middle, and lower thirds and nasal root as the grade of deviation is indicated.

\section{History \& Legends}

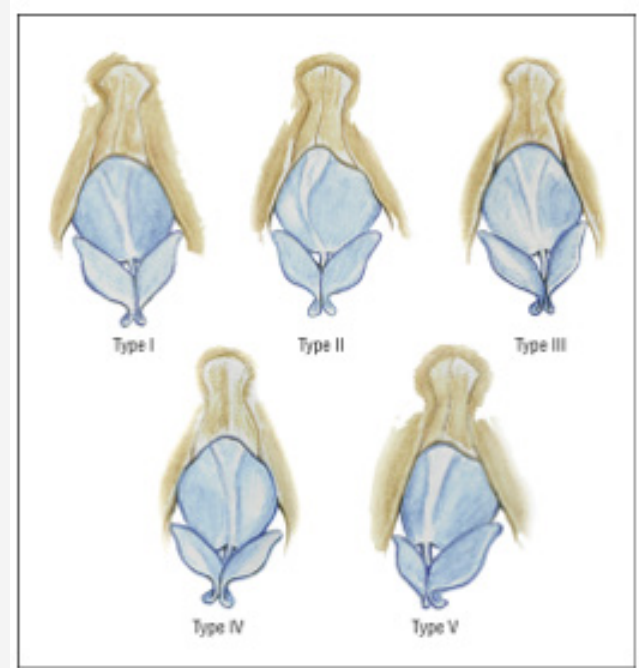

Figure 1: Classification of the deviated nose. For explanation of the 5 types, see the "Classification" subsection of the "Methods" section. "Figure 1".

History and classification: The only classification in the English publishes is "Yong Ju Jang; Jong Hwan Wang; Bong-Jae Lee “ published a classification of nasal deviation in 2008 on reference "Arch Otolaryngol Head Neck Surg. 2008;134(3):311-315". They classified the nasal deviation into 5 types: I, a straight tilted bony pyramid with a straight tilted cartilaginous vault in the opposite direction; II, a straight tilted bony pyramid with a concavely or convexly bent cartilaginous vault; III, a straight bony pyramid with a tilted cartilaginous vault; IV, a straight bony pyramid with a bent cartilaginous vault, and $\mathrm{V}$, a straight tilted bony pyramid and a tilted cartilaginous dorsum in the same direction. The classification has never used in facial plastic references for its complexity and the lack of its clinical implication, it was as an observation of seventyfive sample size with twisted noses [1]. The classification talked about the nose as 2 horizontal subunits (the bony pyramid and the cartilaginous vault) with respect to the facial midline which was not clearly identified (Figure 1).

My study divides the external nose, for correction purposes, into three parts; upper, middle and lower. Each could be straight (Grade 0) or deviated first, second or third degree. It presents the nasal root separately because it is either not deviated (Grade 0), or deviated (Grade 1). The classification takes the axial facial midline and its equal distances from the brow-tip lines as the norm.

The Classification helps at drawing a more precise picture of the outlining of the nasal deviation, helps at quantifying the improvement or the clearance of the deviation after surgery, it gives the surgeon an idea about the expected surgical time and could help at standardizing the number of the available techniques for correcting each grade of each third of the nasal deviation. 


\section{Nasal Parts and its Aesthetic Lines}

The external nose is divided into 3 parts; upper, middle and lower thirds [2]. The upper is a bony structure made from the nasal spine of the frontal bone, two nasal bones, and the ascending or frontal process of the maxilla on both sides. The Middle third is a cartilaginous extension of the nasal dorsum consists from a combination of triangular septal cartilage on the middle, the upper alar cartilages on each side and little sesamoid cartilages on the basal connection with the nasal bony pyriform aperture and the lower attachment of the alar base. The lower third is a fibrofatty cartilaginous structure with a condensation of retaining ligaments, it is structured by the lower alar cartilages, caudal part of the nasal septum and the anterior maxillary nasal spine.

Alar base stabilization and the anterior nasal spine suture technique are essential for achieving a stable midline anchor for ensuring long-term straight nasal alignment.

The dorsal aesthetic line [3] (DAL), provides a thoroughfare between the radix and nasal tip, it is in the middle between the two brow-tip aesthetic lines (BTAL) (Figure 2).

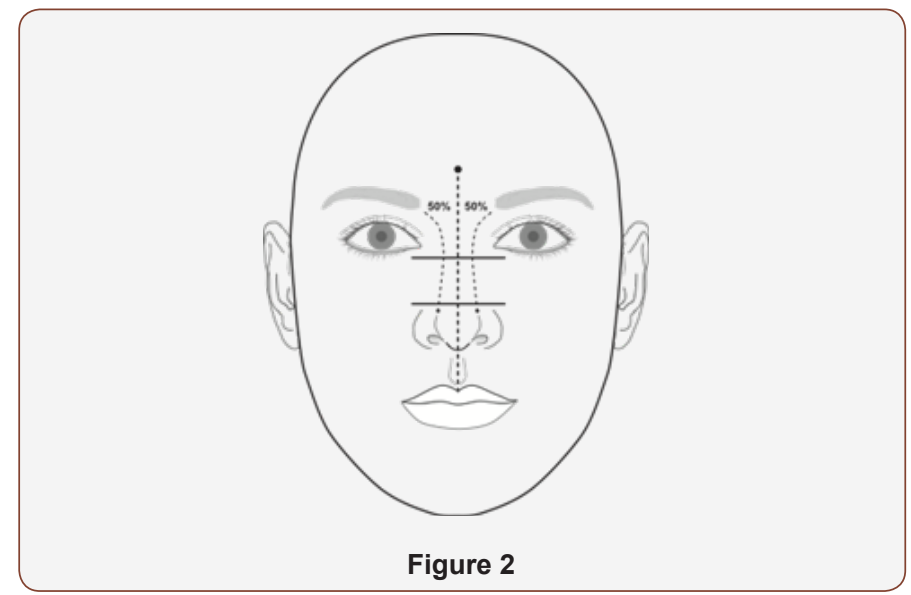

\section{Brow-Tip Aesthetic Lines}

Each line follows a slight curve from the medial aspect of the superior orbital rim at the medial brow, passing through the lateral radix and extending to the nasal tip defining points. Brow-tip aesthetic lines are expected to follow an unbroken path [4].

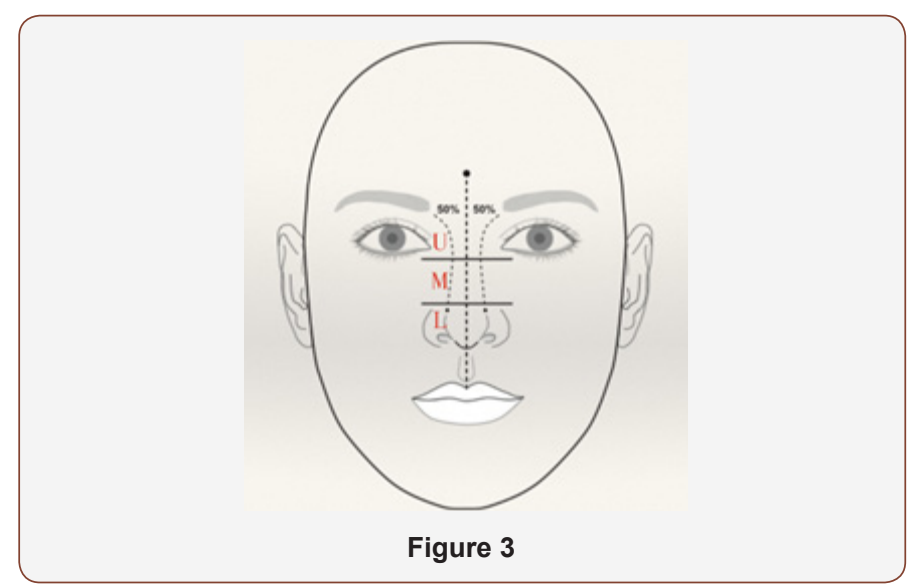

The BTALs form a subtle and fluid hourglass appearance, slightly wider at the radix and tip, with the narrow most portions in the middle third (Figure 3).
During rhinoplasty, the facial plastic surgeon must carefully recreate a smooth brow-tip contour for a natural and aesthetically pleasing result $[5,6]$.

Assessment of the dorsal aesthetic lines is helpful for identifying dorsal irregularities that will need to be corrected. These include asymmetry, excessive width, excessive narrowness or poor definition, likewise inverted $\mathrm{V}$ deformity, which results from a disruption of the brow-tip aesthetic lines secondary to prominent caudal edges of the nasal bones and loss of mid vault support and could hide a nasal dorsal aesthetic asymmetry.

\section{Deformities of the Brow-Tip Aesthetic Lines "BTALs"}

Irregularity: It could reflect an underlying asymmetry. Rasping, dice cartilage with Tissel glue, camouflage facial graft, adipose tissue-derived stem cells (ADSCs), or dermal fillers injection give the ideal solution as per case.

Spacing: The BTALs are at equal distance from the facial axial midline but they are more away from each other or do not fit the standard hourglass feature. We see such deformities after hump reduction without lateral osteotomies in some cases, thick nasal bones and/or thick central ethmoidal block (CEB), congenital nasal deformities, and postcentral nasal fracture. Broad and thick unilateral or bilateral spreader flap/graft could show the spacing deformity in the mid vault. Sharp pointed boxy tip as a congenital or iatrogenic deformity is another cause for the spacing deformity.

Convexed deformity: Both BTALs are at equal distance from the midfacial axial line, but they are both over-projected. It reflexes over-projected nasal dorsum in the majority of cases [7-9].

Concaved deformity: Both BTALs are at equal distance from the midfacial axial line, but they are under-projected either in the boney part, in the middle or the lower thirds. The former reflects under-projected nasal bony dorsum, the second reflects saddling deformity or supra-tip depression, the lower third depression could reflect droopy tip or Polly-beak deformity.

Washed-out: When the BTALs cannot be detected in a flat nasal dorsum.

Twisted nose: When the BTALs are at unequal distances from the midfacial axial line. The deviation could be at the level of upper, middle or lower thirds and could be at 2 or 3 thirds but valiant or equal in the severity depending on the shape of the crooked nose $[10,11,12,13]$.

\section{Classification and quantification of Nasal Deviation}

For the purpose of clinical implication, the nose is divided into three parts as aforementioned before. When the Nasal Root at the level of radix is not on the midline, it needs to be separately mentioned.

Ro: Nasal root.

U: Upper bony third.

M: Middle inner nasal valve cartilaginous third.

L: Lower external nasal valve and alar third. 
Midline of the nose: is identified the vertical or sagittal line connects between the mid-brow glabellar point and a mid-filtrum point on no-congenitally deformed upper lip(Table 1 ).

Table 1:

\begin{tabular}{|c|c|c|c|c|}
\hline & Nasal Root & Upper Third & Middle Third & Lower Third \\
\hline Grade 0 & Ro & U 0 R/L & M 0 R/L & L 0 R/L \\
\hline Grade I & Ro I & U 1 R/L & M 1 R/L & L 1 R/L \\
\hline Grade II & N/A & U 2 R/L & M 2 R/L & L 2 R/L \\
\hline Grade III & N/A & U 3 R/L & M 3 R/L & L 3 R/L \\
\hline
\end{tabular}

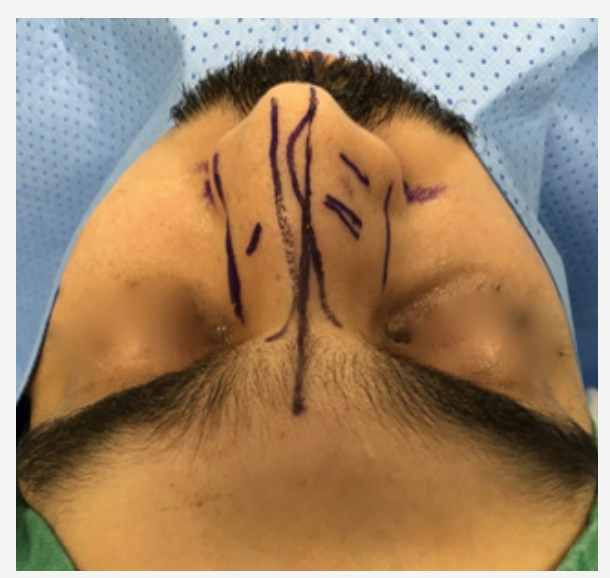

U1R, M2R \& L3R.

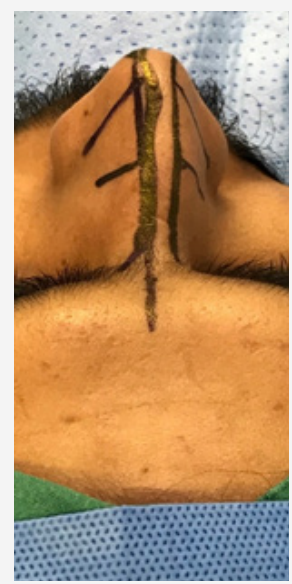

URo1R, U3R, M3R \& .L3R.

Figure 4

Grade (2) nasal deviation: One of the BTALs touching the axial facial midline (Figure 5)

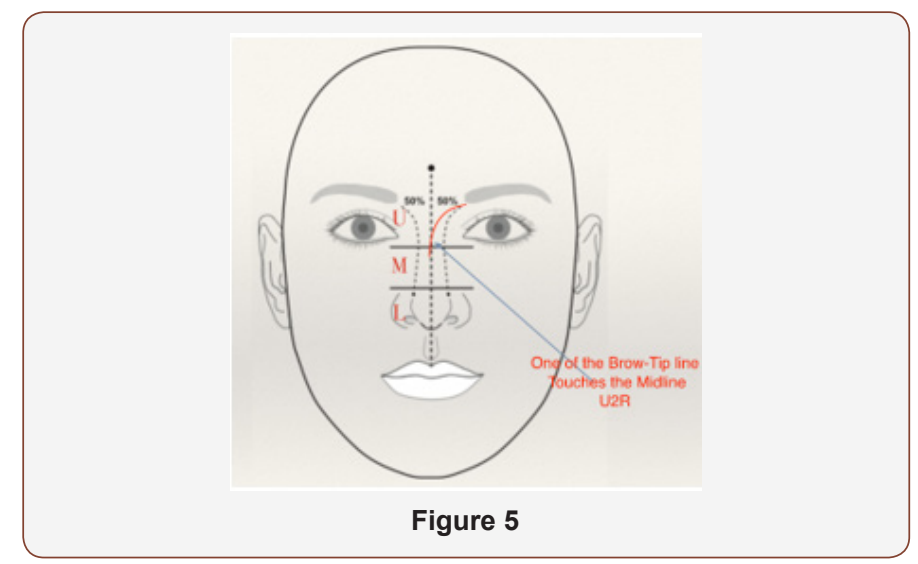

Figure 5

Brow-Tip Aesthetic line: There are two lines each one is a curvilinear line extends from the medial aspect of the brow at the level of the medial border of the upper orbital margin extending down the lateral aspect of the radix and the dorsal projection of lateral nasal sidewall to the tip defining point at the side of the line.

Grade (0) nasal deviation: each BTAL is $50 \%$ away from the midline (Figure 3).

Grade (1) nasal deviation: the BTALs are unequally away from the midline but none of them touching the midline (Figure 4)

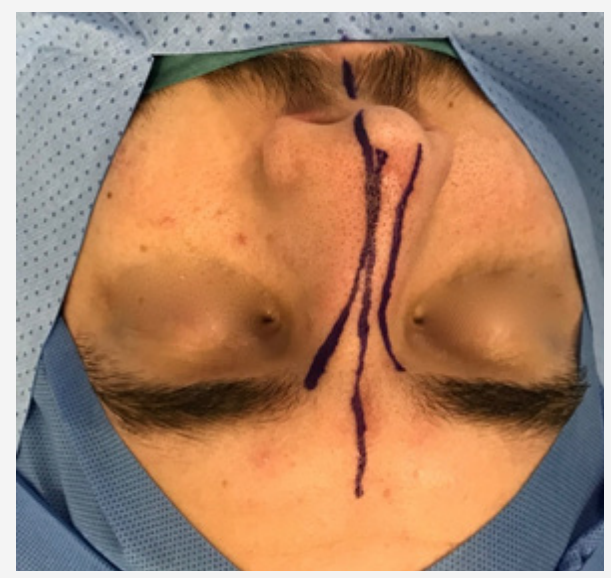

U1R, M2R \& L3R.

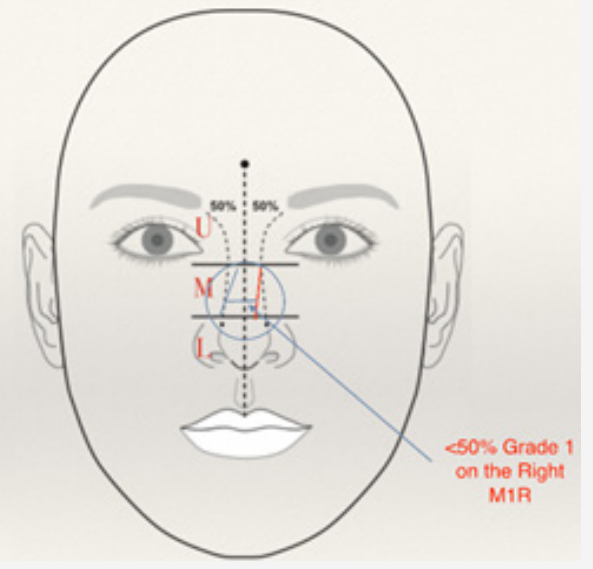

Grade (3) nasal deviation: One of the BTALs is passing over and crossing the axial facial midline (Figure $6 \& 7$ )

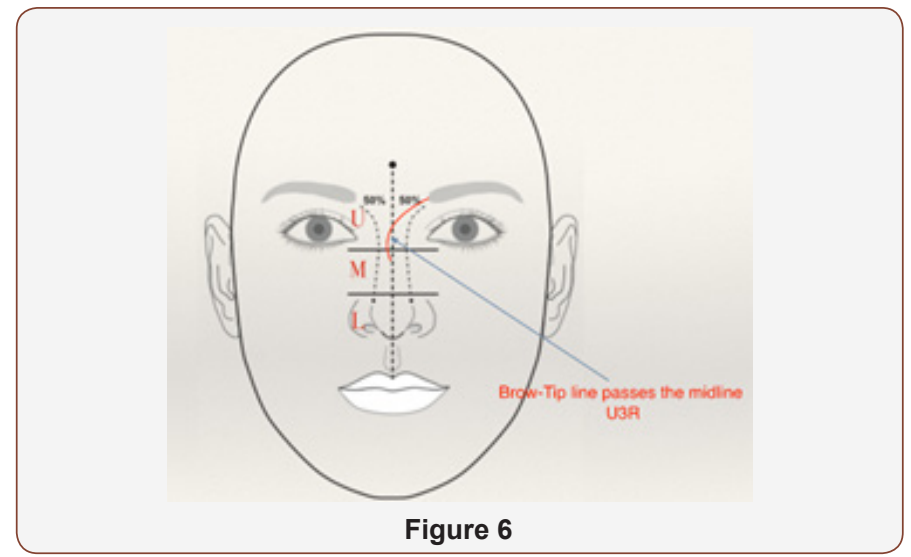

Citation: Abdul RS Juratli. Classification of Nasal Deviation. Online Journal of Otolaryngology and Rhinology. 1(2): 2019. OJOR.MS.ID.000509. DOI: 10.33552/OJOR.2019.01.000509. 


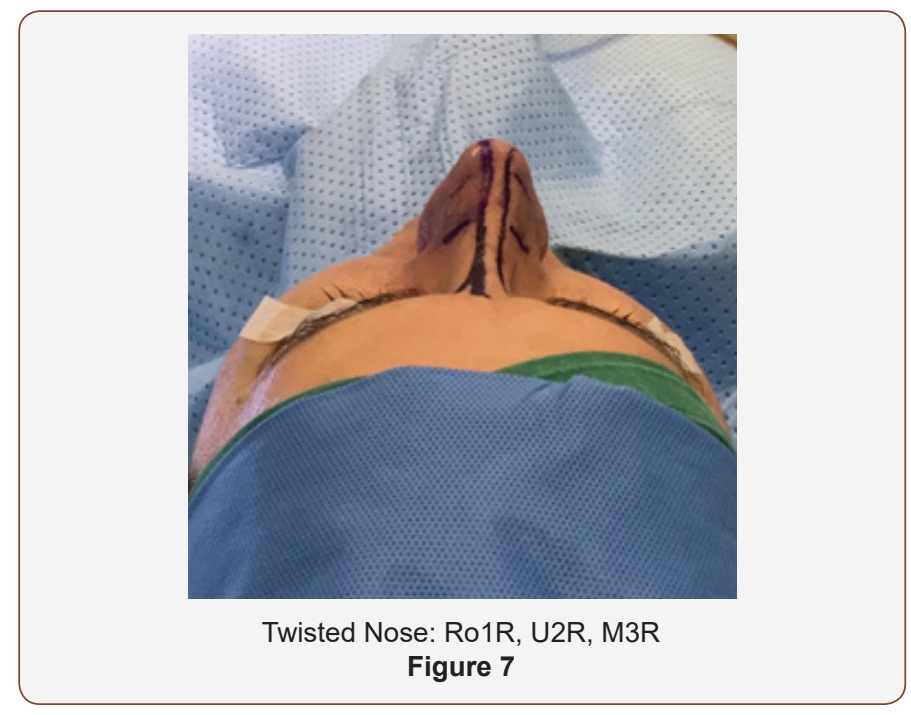

The classification is applied for pre and post op assessment and for the nose which has a history of any surgery. We need to mention any irregularity alongside the brow-tip lines separately.

The classification is applied when both BTLs touch each other in such case of having a sharp, edgy nasal dorsum, but there will be either Grade 0 when the midline meets the effused BTLs or Grade 3 when the midline does not touch the area of effusion (Figure 8).

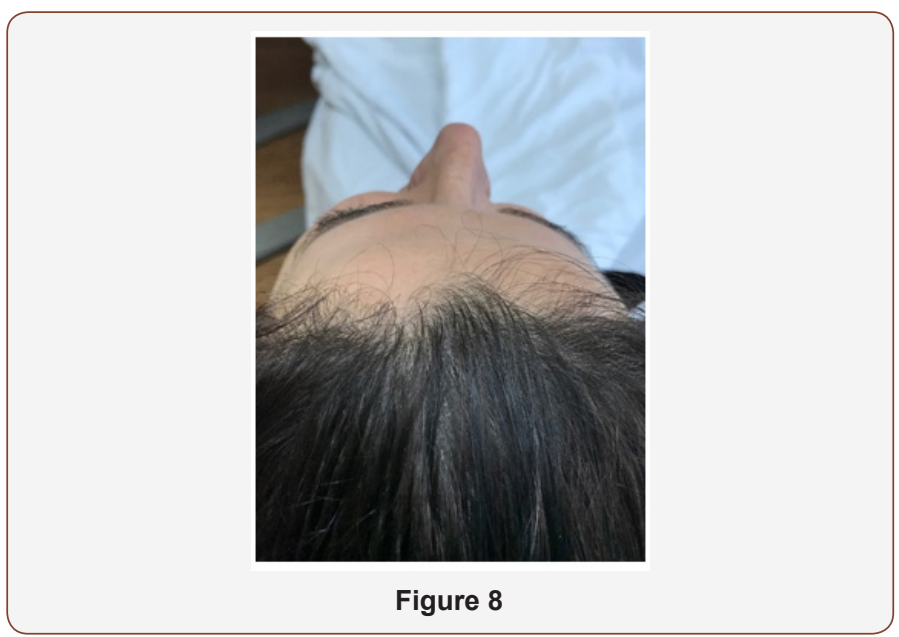

\section{An example of the Final Classification}

Ro 1 R: The BTALs at the level of the root of the nose of the nose are at the unequal distance from the axial midline, but none is touching the midline and the deviation is on the right (Figure 4).

U 2 R: One of the BTALs is touching the midline in the upper third and the deviation is on the right (Figure 5).

M 3 R: One of the BTALs is crossing over the midline in the middle third and the deviation is on the right (Figure 6).

L 3 R: One of the BTALs is crossing over the midline in the lower third and the deviation is on the right

Finally: The nose is out of the midline Ro1R, U2R, M3R, L3R, such deformity is common in Developmentally Deviated Nose (DDN) which often comes with congenital facial asymmetry (Figure $6 \& 9)$

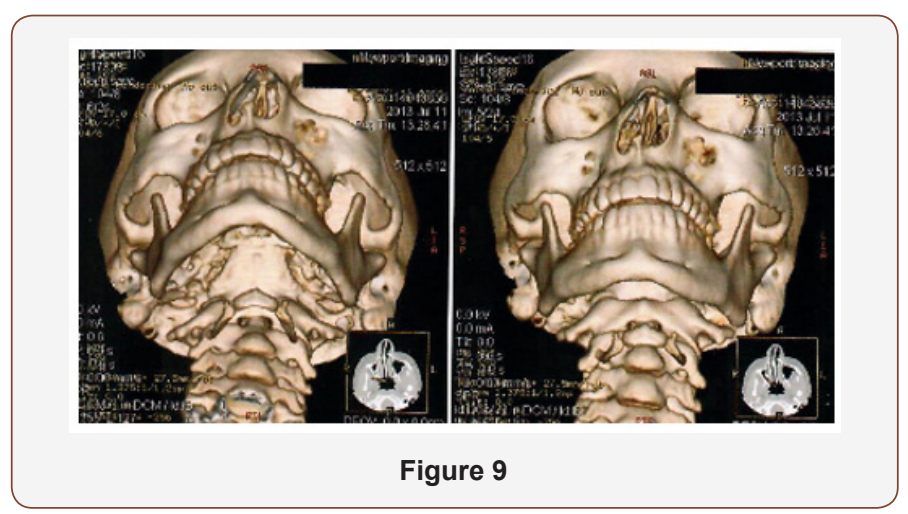

\section{Surgical Implication for the New Classification}

\section{General considerations}

Root deviation (Ro): Consider root transverse osteotomy and fracturing of the frontal nasal spine.

Upper third deviation: Consider tilted hump reduction and central ethmoidal block deviation. Medial, medial oblique, lateral, intermedial and transverse osteotomies are required as per the severity of the deviation. Camouflage nasal dorsum and/or the short concaved side-wall could be essentially required [14-16].

Middle third deviation: Consider thick, thin, unilateral, bilateral or extended spreader graft. Spreader flap is often applied when there is a hump reduction for $3 \mathrm{~mm}$ Hight or more. Clocking suture helps in some cases, and crisscross with or without camouflaging the key-stone junction with diced cartilage and platelets poor plasma (PPP) or Tisseel glue or facia/perichondrium grafts should be accounted to avoid a gap depression at the bonycartilaginous junction. Extracorporeal septoplasty could be the only option to correct a severe deviation in the bony-cartilaginous junction and mid vault of the nose [17].

Lower third deviation: Consider stabilizing the caudal part of the septum on the midline. There are few techniques to stabilize the caudal septum on the anterior nasal spine. Creating straight caudal septal cartilage usually proceed the stabilization, by getting a firm L-Strut, extended septal graft, extended spreader graft or deploying $0.15 \mathrm{~mm}$ thickness, perforated PDS foil plate for a temporary support [18].

Each of the lower alar cartilage domal angle and interdomal angle should be optimized and stabilized with the support of columellar strut, tongue-in-groove or anterior septal angle suspension suture on the midline. The lateral alar crurae should be wide enough, well supported and symmetrical before the final closure [19].

\section{Tailoring Considerations}

Quantification of nasal deviation by giving ascending numbers for each third of external nasal alignment will draw a clear picture for the listener to understand the description of nasal deviation. Specific shapes raises risk on keystone area and a suspicion about Lateral Wall Insufficiency "LWI" and nasal valve deficiency.

How the classification help in managing cases and planning for the most convenient approach? 
M3: Consider unilateral spreader graft, camouflage graft, asymmetrical auto spreader flap, and Clock suture at the same time. Keystone area is at significant risk, and extracorporeal septoplasty or Anterior Septal Reconstruction ASR is a technique which is, thought about in case of dorsal and caudal septal deformity. We could often find ourselves dealing with Zone 1 lateral wall insufficiency LWI.

L1: With straight and robust caudal septum, consider symmetrical intradermal followed by tip suspension on the anterior septal angle or Tongue-in-groove.

L3: with tip ptosis, asymmetrical lateral crural steal sutures and tip suspension with or without columellar strut and Shield graft or diced cartilage in glue and fascial graft should be considered dependent on the skin thickness and the severity of the ptotic tip. In case of over projected tip and tip ptosis, consider asymmetrical lateral crura overlap and vertical cut [20].

Finally: When the situation is Grade 2 in any of the thirds, address something in between as per the third neighbor deformity.

\section{Important Consideration}

The anterior nasal valve integrity is often at risk, supporting the boundaries of the internal and external parts of the valve will give good long-lasting nasal function. Most of the aforementioned techniques will certainly help.

The Developmental Deviated Nose (DDN) often carries alar base level asymmetry which could require grafting the alar base-pyriform fossa junction with allogenous, homogenous or autogenous grafts at the receded side of the alar base.

\section{Acknowledgements}

None.

\section{Conflict of Interest}

No Conflict of Interest.

\section{References}

1. Abdul Juratli (2017) Twisted Nose and Anterior Nasal Valve Plasty Classification You Can Rely On. J Otolaryngol ENT Res 7(2): 00200.

2. Sam P Most, Moubayed SP (2017) Correction Deviation of the Lower Third of the Nose. Facial Plast Surg 33(2): 157-161.
3. Toriumi DM (2017) Dorsal Augmentation Using Autologous Costal Cartilage or 6- Microfat-Infused Soft Tissue Augmentation. Facial Plast Surg 33(2):162-178.

4. Daniel PK (1992) The nasal tip: anatomy and aesthetics. Plastic Reconstructive Surg 899(2): 216-224.

5. Davis R (2006) Rhinoplasty and concepts of facial beauty. Facial Plast Surg 22(3): 198-203.

6. Cobo R, Numa W (2011) Mestizo Rhinoplasty. In: Azizzadeh B, ed. Master Techniques in Rhinoplasty. Philadelphia, PA: Elsevier/Saunders 397-414.

7. Natalie A Krane, Jeffrey D, Moneta LB, Kim MM (2017) Aesthetiics of the nasal dorsum: proportions, light, and shadow. Facial Plastic Surgery nol 33(2): 120-124

8. Hamilton GS, Recker C (2016) Evaluation of the Patient with Nasal Obstruction. Facial Plast Surg 32(1): 3-8.

9. Bilen BT, Tenekeci G (2016) Nasal dorsum management using fragmented cartilage grafts. J craniofac Surg 27(7): 1647-1651.

10. DeRosa J (2016) Does the Nose Have a Function Beyond Breathing? Facial Plat Surg 32(1): 9-16.

11. Aaron M Kosins, Daniel RK, Nguyen DP (2016) Rhinoplasty: The Asymmetric Crooked Nose an Overview. Facial Plast Surg 32(4): 361373.

12. Kutubize A (2015) Nasal aesthetic lines and rhinoplastytechnical tricks. Plast Aesthet Res 2:315-319.

13. Toriumi, Watson (2001) Innovative Surgical Management of Crooked Nose in Rhinoplasty. In: The Dallas Rhinoplasty Symposium Text. ST. Louis, MO: Quality Medical Publishing.

14. Azizzadeh B, Rielly M (2016) Dorsal hump reduction and osteotomies. Clin Plast Surg 43(1): 47-58.

15. Roostaeian J, Unger JG, Lee MR, Geissler P, Rohrich RJ (2014) Reconstitution of the nasal dorsum following component dorsal reduction in primary rhinoplasty. Plast Reconstr Surg 133(3): 509-518.

16. Sharafi M, Jalessi M, Adamson P (2015) Newly designed upper lateral cartilage flap for preventing depression of the keystone area in largenose septorhinoplasty. JAMA Facial Plast Surg 17(6): 399-404.

17. Azizzadeh B, Mashkevich G (2011) Middle Estern Rhinoplasty In: Azizzadeh B, ed. Master Techniques in Rhinoplasty. Philadelphia. PA: Elsevier/Saunders 387-396.

18. Daniel RK (2011) Mastering Rhinoplasty: A Comprehensive Atlas of Surgical Techniques. Stuttgart: Springer 72.

19. Watson, Kridel RW, Kwak ES (2016) Columellar Aesthetics in Open Rhinoplasty. Facial Plastic Surgery 32(4):333-338.

20. Gordon CR, Alghoul M, Golberg JS, Habal MB, Papay F (2011) Diced cartilage graft wrapped in AlloDerm for dorsal nasal augmentation. J Craniofac Surg 22(4): 1196-1199. 